\title{
Experimental aspects of QCD studies at the LHC
}

\author{
S. Tapprogge $\mathrm{e}^{\mathrm{a} *}$ \\ ${ }^{a}$ CERN, EP Division, Geneva
}

The LHC will allow precision tests and measurements of QCD in as yet unexplored kinematic regions. The detailed understanding of QCD is important for almost all physics processes to be studied at LHC, as the production mechanisms are mostly controlled by QCD.

The multi-purpose detectors (ATLAS and CMS) have been optimized for precision measurements within pseudorapidities of $|\eta|<2.5$ and have calorimetric coverage up to $|\eta|=5$. The expected performance will allow precise studies of final states containing electrons, photons, tau's (single hadrons), muons and jets.

This contribution discusses various measurements of QCD processes at LHC, the kinematical reach and the expected statistical uncertainties for selected examples.

\section{Introduction}

The production mechanisms for most processes at LHC will be controlled by QCD and a detailed understanding of these is mandatory for precision measurements, like the mass of the $W$ boson. Furthermore proton-proton collisions at a centreof-mass energy of $\sqrt{s}=14 \mathrm{TeV}$ will represent a regime, where QCD as the theory of strong interactions can be precisely tested at the highest energy. Besides these tests, studies of QCD related processes at LHC will allow for detailed measurements leading to new information on the parton densities of the proton and possibly also measurements of the strong coupling constant, both in as yet unexplored kinematical regions. The transition between perturbative and non-perturbative QCD could be studied using hard diffractive scattering, which poses significant experimental challenges due to the harsh LHC environment.

This report presents an overview of QCD studies at LHC and discusses several related experimental aspects. First, a brief description of the ATLAS and CMS detectors is given, followed by a discussion of minimum bias events and their properties. Next, issues related to hard diffractive scattering are presented together with possible experimental selections of this type of events.

${ }^{*}$ Invited talk at the 'QCD 99 International Euroconference in Quantum Chromodynamics', Montpellier, France, July $7-13,1999$.
Then, the production of jets, photons, Drell-Yan pairs and $W / Z$ bosons and heavy quarks is discussed, together with a description of the possible influence of these measurements on the knowledge of parton densities.

\section{The ATLAS and CMS detectors}

The ATLAS[1] and CMS[2] detectors are the two general purpose $4 \pi$ detectors for LHC. Both detectors will have silicon pixel and silicon strip detectors mounted close to the interaction region, allowing robust tracking and precise vertex measurements. They are followed by a transition radiation tracker in ATLAS and microstrip gas chambers in CMS. In case of CMS, the tracking volume is surrounded by calorimetry (lead tungstate crystals in the e.m. compartment and copper-scintillator sandwich structures for the hadronic part), followed by a $4 \mathrm{~T}$ solenoid. The return yoke of the magnet is instrumented with muon chambers. In case of ATLAS, the tracking detectors are surrounded by a $2 \mathrm{~T}$ solenoid, which is located in front of the calorimetry (liquid Argon (LAr) based for the e.m. part and a combination of scintillator based and LAr based sampling calorimeters for the hadronic part). The muon measurement in ATLAS is performed by an air-core toroid system with muon detectors.

Both detectors have been optimized in terms of lepton (esp. electron and muon), photon, jet and 
missing $E_{T}$ measurements for precision measurements within the Standard Model and searches for the Higgs boson(s) and for physics beyond the Standard Model. The precision measurements of leptons and photons are performed over the range $|\eta|<2.5$, whereas the acceptance for jets and for the measurement of missing $E_{T}$ extends up to $|\eta|=5$.

An additional challenge is to provide an efficient trigger system, which has to reduce an interaction rate of $10^{9} \mathrm{~Hz}$ to about $100 \mathrm{~Hz}$ going to mass storage. Both detectors will have a first level trigger realized in hardware, using information only from the muon systems and the calorimeters (in a coarse granularity). The maximal latency is $2.5 \mu \mathrm{s}$ (ATLAS) and $3 \mu \mathrm{s}$ (CMS). The higher trigger levels will provide flexibility in the algorithms to be executed and offer access to the full granularity data from all sub-detectors, to achieve the necessary rejection from $\mathcal{O}(100) \mathrm{kHz}$ after the first level.

Due to the large number of events available in control samples such as $Z \rightarrow l^{+} l^{-}, \gamma(Z)+$ jet and $W \rightarrow$ jet + jet (from top quark decay), a precise determination of the energy scale for leptons (to an accuracy of better than $0.1 \%$ ) and jets (to an accuracy of $1 \%$ ) will be possible. These uncertainties are going to be in many cases the limiting experimental systematic uncertainties for precision measurements. Measurements of crosssections however will most likely be limited by the accuracy of the measurement of the absolute value of the luminosity; the expected uncertainty is estimated to be about 5-10\%. Studies are ongoing to reduce this value by using precisely calculable reactions (like two photon production of muon or electron pairs), which might allow to reduce the uncertainty on the luminosity measurement to $1-2 \%$.

The results presented in this contribution are mostly from ATLAS (based on [3]), however a quite similar reach and potential is expected for CMS. The differences in the design of the detectors should provide cross-checks on experimental sources of systematic uncertainties. More details on the studies presented here can be found in [3].

\section{Minimum bias events}

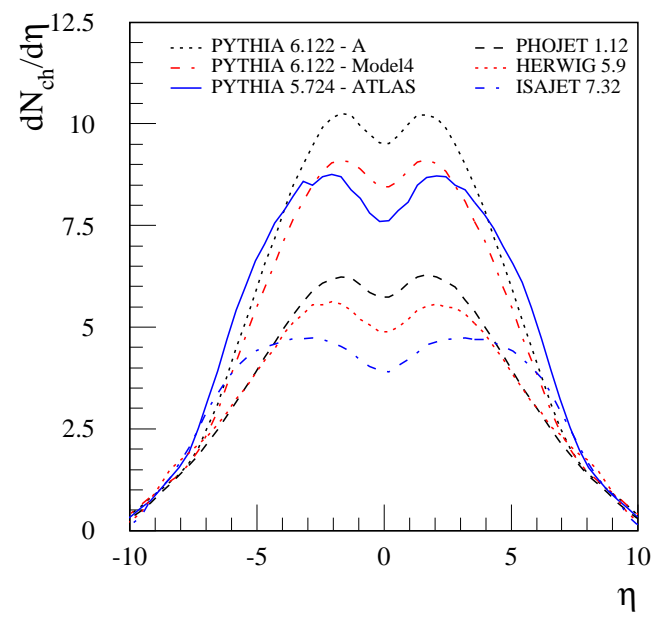

Figure 1. Charged particle density $\mathrm{d} N_{c h} / \mathrm{d} \eta$ as a function of pseudorapidity $\eta$ at LHC as predicted by different models.

A precise knowledge of the properties of minimum bias events is important for running at the LHC design luminosity of $10^{34} \mathrm{~cm}^{-2} \mathrm{~s}^{-1}$. In these conditions, on average 25 inelastic interactions are expected every bunch-crossing (i.e. every $25 \mathrm{~ns}$ ). Within $|\eta|<2.5$ in total about 1000 charged particles are expected from these events. The uncertainties in the present modelling of minimum bias events can be seen in figure 1 , which shows the charged particle density as a function of pseudo-rapidity for different models. The models used (except 'PYTHIA 6.122 A' which overestimates the charged particle density by about one unit) are able to describe available data from Tevatron at $\sqrt{s}=1.8 \mathrm{TeV}$. Various settings of the PYTHIA model are shown, which lead to differences of $\leq 1$ charged particle per unit of pseudo-rapidity. The predictions of PHOJET, HERWIG and ISAJET are generally 


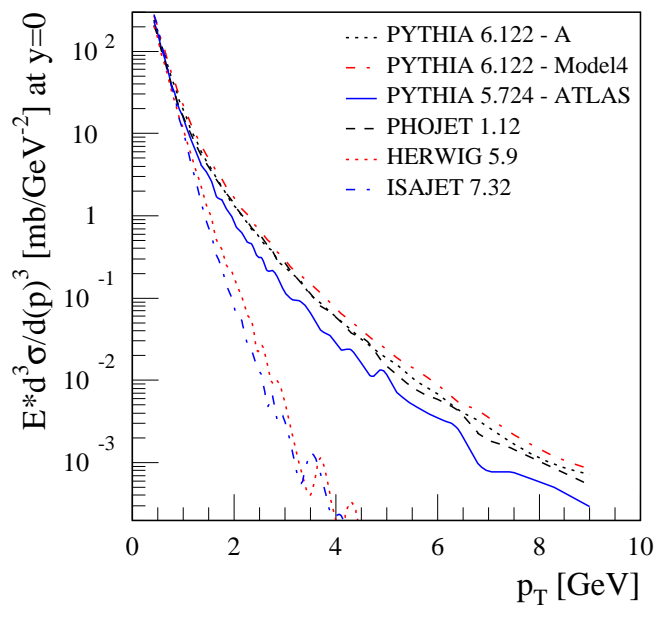

Figure 2. Transverse momentum cross-section $E \cdot \mathrm{d}^{3} \sigma / \mathrm{d} p^{3}$ for charged particles at LHC as predicted by different models.

lower by about $2-3$ charged particles per unit of pseudorapidity. These differences are also present at $\sqrt{s}=1.8 \mathrm{TeV}$, however their magnitude is reduced. It should be noted, that for HERWIG and ISAJET no attempt was made to include contributions from hard scattering (as done in the case of PYTHIA and PHOJET). This is clearly seen in figure 2, where the cross section for charged particle production (at central rapidity $y=0$ ) is shown as a function of the transverse momentum $p_{T}$ of the particle. HERWIG and ISAJET predict a too steeply falling spectrum (this also holds at $\sqrt{s}=1.8 \mathrm{TeV}$, where data are not described by these two models).

Not only the single particle production needs to be measured and understood, also the structure of low $E_{T}$ jets ('mini-jets') is important. For several hard scattering processes a veto on additional jet activity is going to be used to enhance the signal and suppress background contributions.

\section{Hard diffractive scattering}

One of the characteristic signatures of diffractive scattering in hadron-hadron collisions is the appearance of one (or both) of the initial state hadrons in the final state with a momentum close to the beam momentum (so called 'leading hadron').
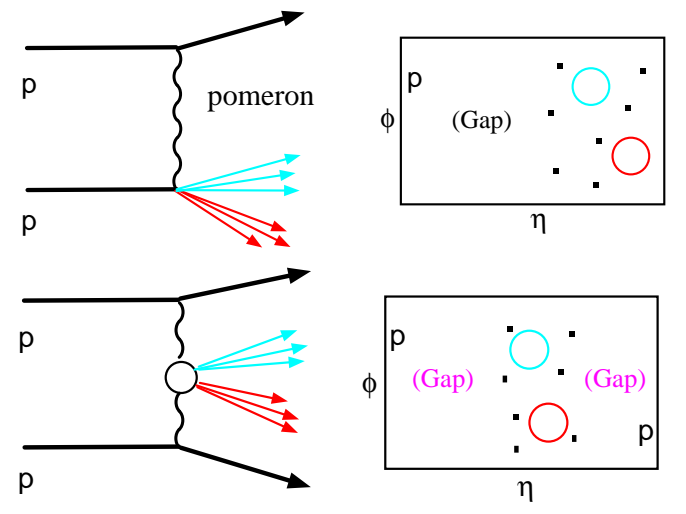

Figure 3. Single hard diffractive scattering (upper plot) and central hard diffractive scattering (lower plot). For both cases, the phase space in $\eta-\phi$ is also shown.

As first observed by the UA8 collaboration [4], events of diffractive nature can contain a hard scattering (leading to production of jets). In case of single diffractive scattering $(p p \rightarrow p+$ $j e t(s)+X$, as shown in the upper part of figure 3$)$ and of central diffractive scattering $(p p \rightarrow$ $p+j e t(s)+X+p$, as shown in the lower part of figure 3 ), the final state is characterized further by the appearance of one (or two) rapidity gaps. These regions in the $\eta-\phi$ space without particle production separate the leading proton(s) from the system $X+j e t(s)$.

In figure 4 the cross-section for di-jet production in single diffractive scattering is shown as a function of the jet pseudo-rapidity $\eta^{j e t}$. One leading proton (at positive pseudorapidity with 


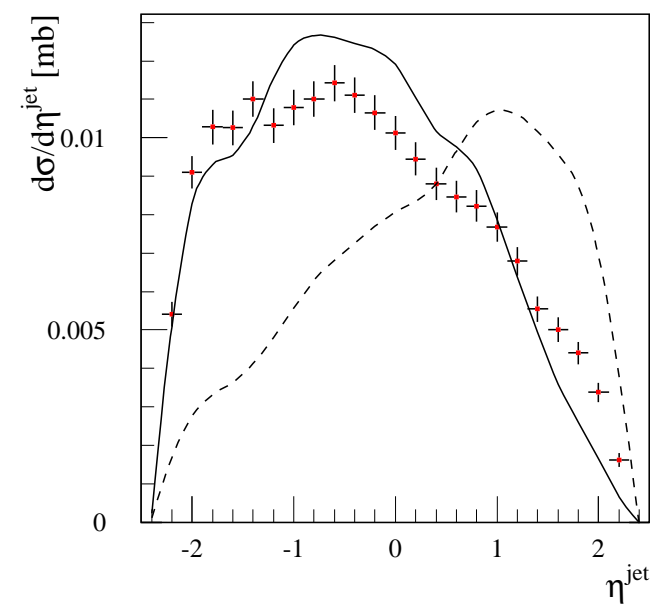

Figure 4. Cross-section for di-jet production in single diffractive scattering as a function of the jet pseudo-rapidity $\eta^{\text {jet }}$. Three different model of the Pomeron structure are used: CKMT (points), 'soft' gluon (solid line) and 'hard' gluon (dashed line).

$x_{F}>0.9$ and $0.01<|t|<1 \mathrm{GeV}^{2}$ ) and at least two jets $\left(|\eta|<3.2\right.$ and $\left.E_{T}>10 \mathrm{GeV}\right)$ are required. Three assumptions have been used for the partonic structure of the Pomeron. They lead to different shapes of the pseudo-rapidity distribution. This indicates that a measurement of single diffractive di-jet production should allow to constrain the Pomeron structure. More studies are needed to quantify the possible kinematic reach and to investigate the effects of the expected breaking of hard scattering factorization in hadron-hadron collisions (which might inhibit an extraction of parton densities) and the effects of the underlying event on the detection in case of a rapidity gap signature.

Figure 5 shows the cross-section for di-jet production in central diffraction as a function of the jet pseudo-rapidity $\eta^{\text {jet }}$. In this case two leading protons (both with $x_{F}>0.9$ and $0.01<|t|<$ $\left.1 \mathrm{GeV}^{2}\right)$ and at least two jets $(|\eta|<3.2$ and

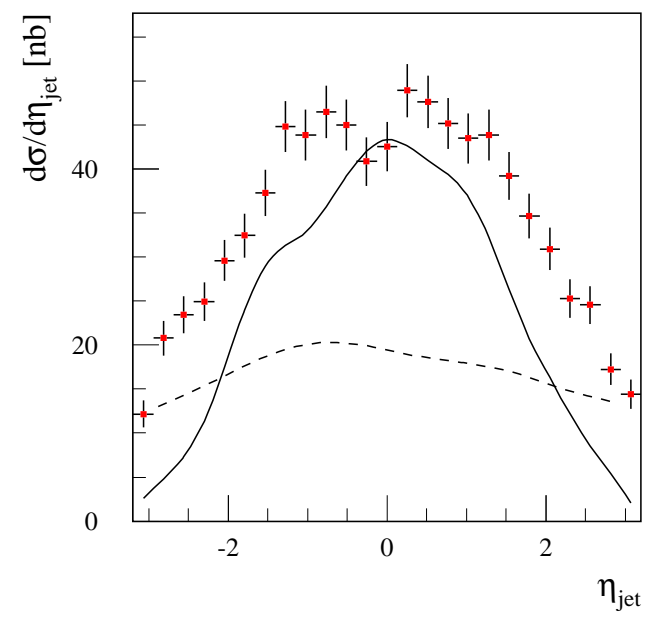

Figure 5. Cross-section for di-jet production in central diffractive scattering as a function of the jet pseudo-rapidity $\eta^{\text {jet }}$. Three different model of the Pomeron structure are used: CKMT (points), 'soft' gluon (solid line) and 'hard' gluon (dashed line).

$E_{T}>10 \mathrm{GeV}$ ) have been required. Also here the shape differs for the three assumptions on the partonic content of the Pomeron. An advantage of central diffraction is the much reduced influence of underlying events and of soft colour exchange, as both protons remain intact. However the expected cross-section is significantly lower.

It is planned by the TOTEM collaboration [5] to install in the CMS interaction region (IR5) additional detectors for the tagging of charged particle production (in the forward direction within $3<|\eta|<7$ ) and for the tagging and measurement of leading protons (Roman Pot systems more than $100 \mathrm{~m}$ away from the interaction point). The combination of the information from these detectors with signatures of hard scattering processes inside the CMS detector should allow several studies of hard diffractive scattering. The ATLAS collaboration is investigating the possibility of installing similar detectors. Such a com- 
bination of detectors could also be used for a measurement of the absolute luminosity. More detailed studies are in progress to quantify the reach in the various kinematic variables and to assess possible running scenarios.

\section{Jet physics}

The production of jets will allow probing the smallest distance scales at the LHC, due to the huge cross-section in comparison e.g. with direct photon and $W / Z$ boson production.

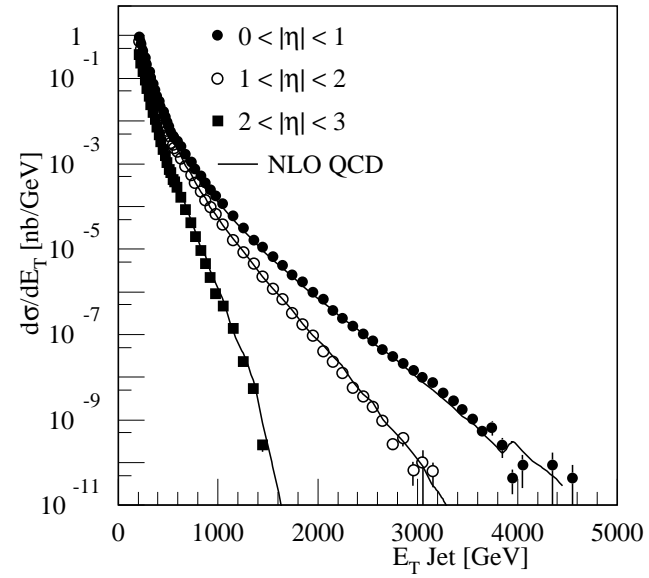

Figure 6. Inclusive jet cross-section as a function of $E_{T}^{j e t}$ of the leading jet for various pseudorapidity ranges.

Figure 6 shows the expected inclusive jet crosssection as a function of $E_{T}$ for three ranges of pseudo-rapidity (from central to forward). The error bars correspond to the expected statistical uncertainty for an integrated luminosity of $300 \mathrm{fb}^{-1}$. For only $30 \mathrm{fb}^{-1}$, about $4 \cdot 10^{5}$ events are expected with $E_{T}>1 \mathrm{TeV}$, about $3 \cdot 10^{3}$ events with $E_{T}>2 \mathrm{TeV}$ and about 40 events with $E_{T}>3 \mathrm{TeV}$.

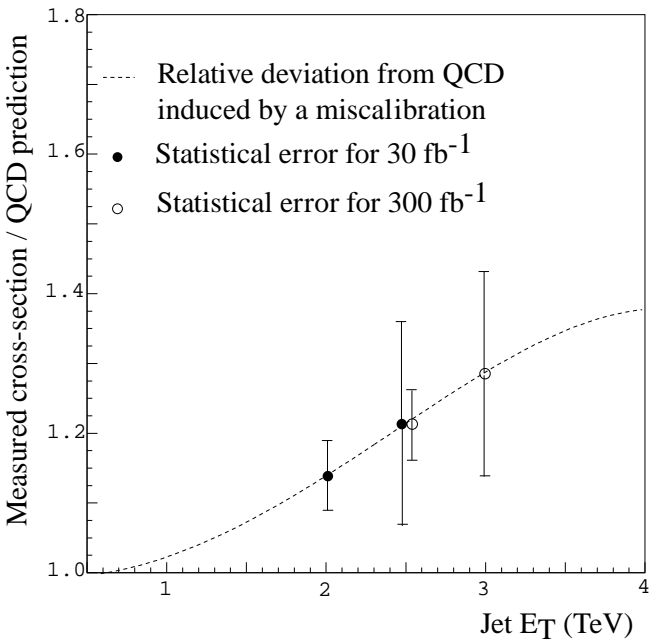

Figure 7. Expected deviation from the QCD expectation for the single inclusive jet cross-section in case of a miscalibration of the calorimeters (see text).

Especially in the region of $E_{T}>1 \mathrm{TeV}$ the dominating (systematic) uncertainty will be given by the knowledge of the jet energy scale. Uncorrected non-linearities of the calorimeters could easily fake a deviation from the QCD expectation, which might be interpreted as a signal of new physics (e.g. of compositeness). In figure 7 the relative deviation from the QCD expectation is shown for the single inclusive jet crosssection as a function of $E_{T}$, in the presence of a miscalibration. The example chosen assumed an overestimation by 0.2 of the degree $e / h$ of noncompensation in the calorimeters (nominal value: $e / h=1.3$ in case of the Tile calorimeter). Also shown are the expected statistical errors on the cross-section for $30 \mathrm{fb}^{-1}$ and $300 \mathrm{fb}^{-1}$ at two values of $E_{T}$. The expected statistical uncertainty is smaller than the effect due to the miscalibration used here.

The measurement of the jet production crosssection is sensitive both to the quark and gluon densities. The contribution due to gluons de- 


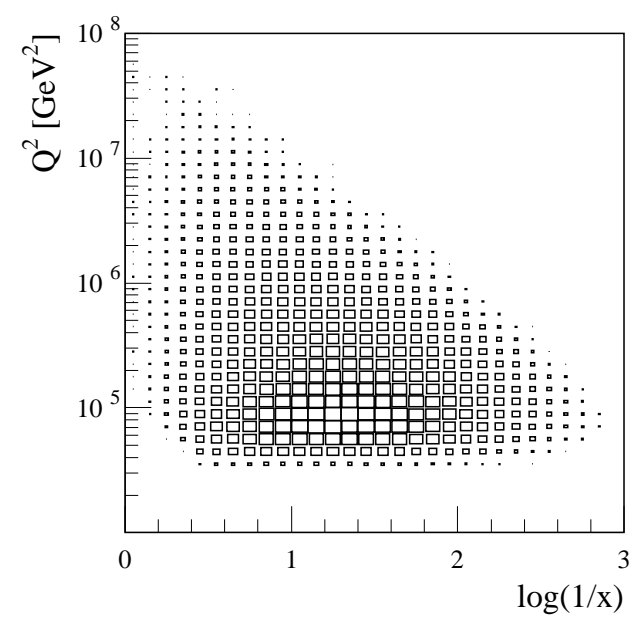

Figure 8. Reach in the $\left(1 / x, Q^{2}\right)$ plane from di-jet production.

creases with increasing $E_{T}$. A measurement of di-jet production (pseudorapidities $\eta_{1,2}$ of the two leading jets and minimal $E_{T}$ for both jets) can be used to reconstruct the parton momenta $x_{1,2}$ entering the hard scattering and the scale $Q^{2}$ of the hard scattering via (at leading order):

$x_{1,2}=\frac{E_{T}}{\sqrt{s}}\left(e^{ \pm \eta_{1}}+e^{ \pm \eta_{2}}\right)$,

$Q^{2} \approx 2 E_{T}^{2} \cosh ^{2} \eta^{*}\left(1-\tanh \eta^{*}\right)$,

where $\eta^{*}=0.5 \cdot\left|\eta_{1}-\eta_{2}\right|$. In figure 8 the reach in $\left(1 / x, Q^{2}\right)$ is shown for di-jet events with $E_{T}>180 \mathrm{GeV}$ and $|\eta|<3.2$. Only those bins which contain more than 100 events (for $300 \mathrm{fb}^{-1}$ ) are visible. A wide range in Bjorken $x$ is covered for large scales (e.g. $0.01<x<0.6$ for $\left.10^{5}<Q^{2}<10^{6} \mathrm{GeV}^{2}\right)$. This has to be compared to the kinematical limit of HERA in $Q^{2}$ of about $10^{5} \mathrm{GeV}^{2}$, which is reached only for large values of $x$. More studies are needed to quantify the accuracy expected on the extraction of parton densities based on jet cross-section measurements. In addition, a combined extraction of par- ton densities based on additional processes (from LHC data alone - as discussed in the following sections - and/or from other measurements - e.g. deep-inelastic scattering) has to be investigated.

Not yet studied in detail is a possible measurement of the strong coupling constant from the ratio of 3 -jet to 2 -jet production. The necessary calculation of the 3-jet cross-section at NLO became recently available. Compared to an extraction of $\alpha_{s}$ from the inclusive jet cross-section, this approach should lead to reduced correlations between the coupling constant and the parton densities (esp. the gluon density).

\section{Photon production}

The direct production of photons can provide sensitivity to the gluon density in the proton, via the QCD Compton graph: $q g \rightarrow q \gamma$. Background processes are $q \bar{q} \rightarrow g \gamma$ and contributions from fragmentation into photons $(q \rightarrow q \gamma)$. In addition, the production of jets containing a leading $\pi^{0}$ can lead to fake photon signatures.

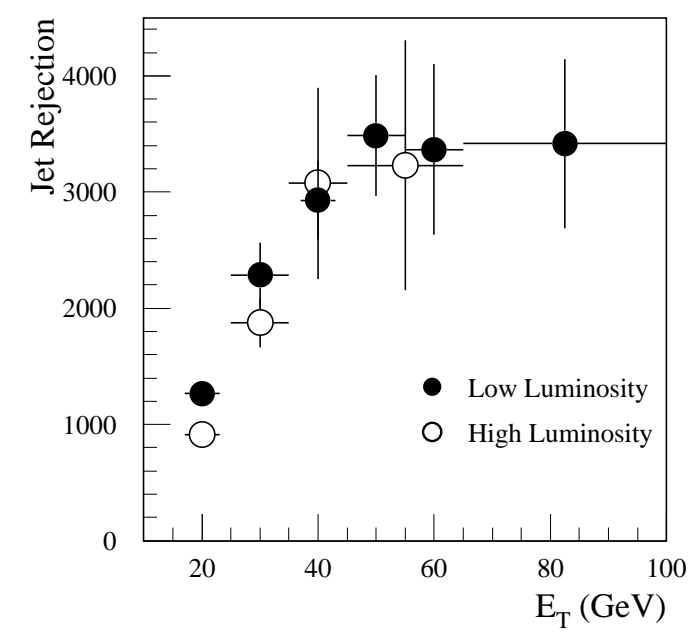

Figure 9. Rejection against jets as a function of the transverse energy $E_{T}$ for a photon identification efficiency of about $80 \%$. 
The experimental challenge is to achieve a good efficiency for photons while providing an excellent rejection against jets. The ratio of the inclusive jet cross-section to the direct photon crosssection is about $10^{3}$ (for $|\eta|<0.7$ and $100<$ $\left.p_{T}<500 \mathrm{GeV}\right)$. Studies done in the context of $H \rightarrow \gamma \gamma$ searches have shown, that efficiencies of about $80 \%$ can be obtained for $p_{T}>40 \mathrm{GeV}$, together with a rejection against jets of about $3 \cdot 10^{3}$. Figure 9 shows the dependence of the jet rejection on the transverse energy $E_{T}$ of the jet. In order to achieve the above-mentioned efficiency, conversions in the material of the inner detector have to be recognized and reconstructed (the probability for a conversion to occur is between $20 \%$ and $40 \%$, depending on $\eta$ ).

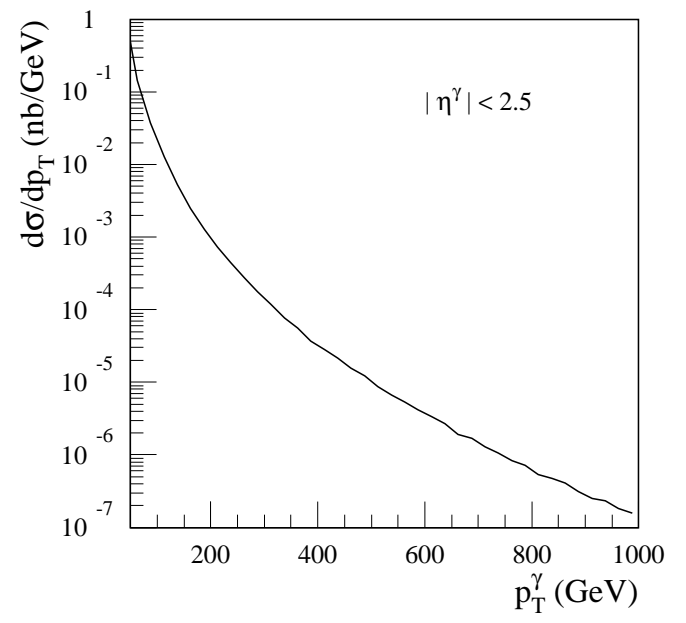

Figure 10. Cross-section for direct photon production as a function of the photon transverse momentum $p_{T}^{\gamma}$.

In figure 10 the cross-section for direct photon production is shown as a function of the photon transverse momentum $p_{T}^{\gamma}$, as obtained from a leading order calculation. For $30 \mathrm{fb}^{-1}$ more than $2 \cdot 10^{4}$ events are expected having a direct photon with $p_{T}^{\gamma}>500 \mathrm{GeV}$. The hard scattering scale is given at leading order by the square of the transverse momentum of the photon $\left(Q^{2}=\left(p_{T}^{\gamma}\right)^{2}\right)$. A minimal $p_{T}^{\gamma}$ of $40 \mathrm{GeV}$ for direct photons corresponds to $Q^{2}>10^{3} \mathrm{GeV}^{2}$ and together with the acceptance in pseudo-rapidity of $|\eta|<2.5$ implies a lower limit on Bjorken $x$ of $x>5 \cdot 10^{-4}$. The upper limit on $x$ depends on the maximal transverse momentum of the photon and thus is given by the available statistics. For a value of $500 \mathrm{GeV}$ the upper limit is $x<0.2$. This kinematic coverage on the gluon density can be compared to HERA, where for $Q^{2}>10^{3} \mathrm{GeV}^{2}$ only the range $x>0.01$ is kinematically accessible.

Events containing a direct photon together with a jet, which are balancing in transverse momentum, may be used for a calibration of the jet energy scale, provided the photon energy scale is known sufficiently well and the background from QCD jet production (leading to fake photon signatures) is well understood.

\section{Drell-Yan and $W / Z$ production}

The production of $W$ and $Z$ bosons at LHC will provide (using the leptonic decays into electrons or muons) a huge sample of events with a clean experimental signature.

For Drell-Yan production of muon pairs about $10^{4}$ events are expected for $30 \mathrm{fb}^{-1}$, which contain a muon pair with an invariant mass $m_{\mu \mu}$ larger than $400 \mathrm{GeV}$. Compared to $W$ and $Z$ production, Drell-Yan pair production allows exploring a larger range in the scale $Q^{2}$ which is given by $Q^{2}=m_{\mu \mu}^{2}$. For $m_{\mu \mu}>400 \mathrm{GeV}$, the range of $Q^{2}>1.6 \cdot 10^{5}$ is covered, with a range in $x$ of $2.3 \cdot 10^{-3}<x<0.34$ (for $|\eta|<2.5$ ).

For the same integrated luminosity, about $10^{5}$ events are expected which contain a $W$ with $p_{T}^{W}>400 \mathrm{GeV}$ (decaying leptonically to electron or muon and a neutrino). The expected number of $Z$ bosons is smaller by about one order of magnitude. In case of centrally produced $W$ bosons ( $W$ boson rapidity $y^{W}=0$ ), the two partons have identical $x$ values of $x_{1,2}=6 \cdot 10^{-3}$. The acceptance limit for the leptons of $|\eta|<2.5$ leads to a lowest $x$ value of about $3 \cdot 10^{-4}$ and a highest $x$ value of about 0.1 . The range in 


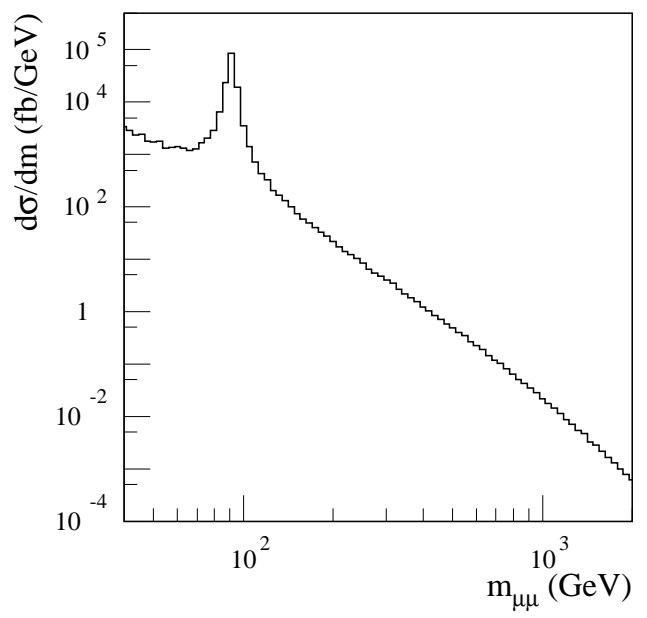

Figure 11. Cross-section for Drell-Yan muon pair production as a function of the muon pair invariant mass $m_{\mu \mu}$.

Bjorken $x$ from $W(Z)$ production is thus given by: $3 \cdot 10^{-4}<x<0.1$ at $Q^{2} \approx 6(8) \cdot 10^{3} \mathrm{GeV}^{2}$. For comparison, measurements of deep-inelastic scattering at HERA with $Q^{2}>10^{4} \mathrm{GeV}^{2}$ are kinematically restricted to the region of $x>0.1$.

The distribution in rapidity $y^{W}$ of the $W$ boson is different for $W^{+}$and $W^{-}$production, as can be seen in figure 12. This difference reflects the differences in the parton distributions leading to the production of a $W: u \bar{d} \rightarrow W^{+}$resp. $d \bar{u} \rightarrow W^{-}$. The average momentum of a valence $d$ quark is smaller than the one of a valence $u$ quark, and as there are two valence $u$ quarks in the proton (w.r.t. to one valence $d$ quark) more $W^{+}$bosons will be produced in the forward direction than $W^{-}$bosons. This difference in the shape of the rapidity distribution survives in the pseudorapidity distribution of the decay leptons, as shown in figure 13. It can be used to constrain the quark and anti-quark densities in the proton.

Given the large statistics expected for $W(Z)$ production and their clean signatures, it might be possible to use these processes for a precise deter-

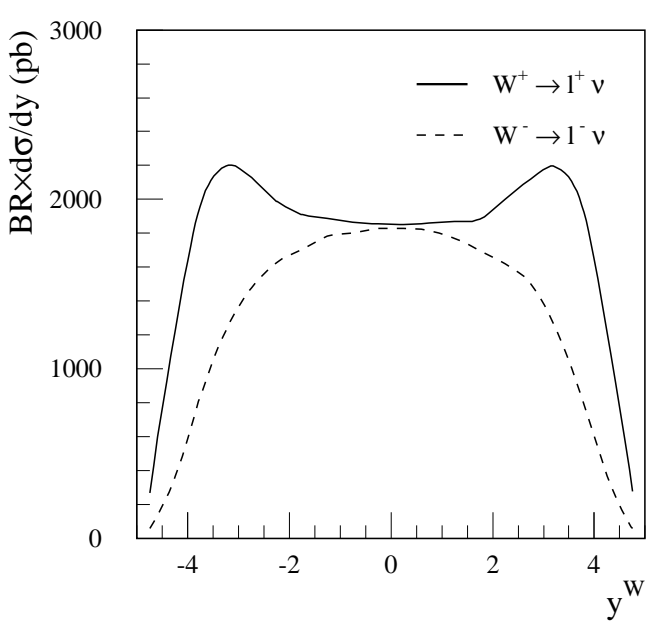

Figure 12. Cross-section for $W^{+}$and $W^{-}$production as a function of the boson rapidity $y^{W}$, including the decay $W \rightarrow l \nu$, where $l=e, \mu$.

mination of the parton-parton luminosity, avoiding the necessity of a difficult absolute luminosity measurement. The idea is to normalize all crosssections to the well measured $W$ cross-section. This method offers the potential to achieve an experimental accuracy at the level of a few percent. More studies are needed however to quantify the expected theoretical uncertainty on the ratio of these cross-sections, which would need to be known at the per cent level.

All cross-sections shown up to now were based on leading order calculations. In many cases, next-to-leading (NLO) calculations are available. The cross-section increases in many cases when NLO corrections are included. This is often due to the opening of additional channels, which only appear at NLO. An example of the size of this increase is shown in figure 14, where the crosssection for $W^{+} W^{-}$production is shown as a function of the missing transverse momentum $p_{T}^{\text {miss }}$. For large $p_{T}^{\text {miss }}$, the cross-section increases at NLO by more than one order of magnitude. 


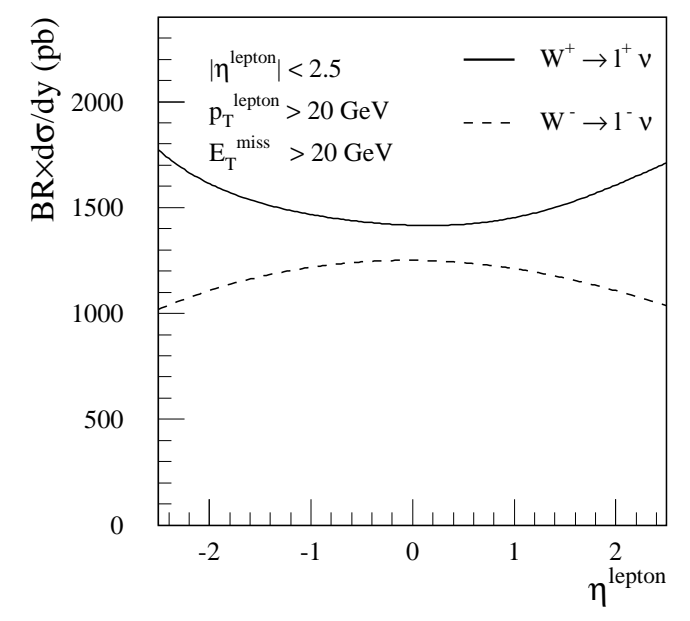

Figure 13. Cross-section for $W^{+}$and $W^{-}$production as a function of the decay lepton pseudorapidity $\eta^{\text {lepton }}$, where lepton $=e, \mu$.

\section{Heavy quark production}

The production of heavy quarks $(b$ and $t)$ proceeds at LHC energies mostly via gluon-gluon fusion. Measurements of e.g. $b$ production could thus provide constraints on the gluon density. However, discrepancies between $b$ production at Tevatron and theoretical predictions have been observed and need to be understood. The predictions are presently about a factor of two smaller than the data.

In figure 15 the cross-section for heavy quark pair production is shown as a function of the minimal transverse momentum $p_{T}^{Q}$ of the heavy quark. For large values of $p_{T}^{Q}(>50 \mathrm{GeV})$, the difference between charm and bottom quark production is negligible (the quark mass being much smaller than the hard scale $p_{T}^{Q}$ ). In the case of $t \bar{t}$ production, a sizeable difference persists up to $p_{T}^{Q} \approx 600 \mathrm{GeV}$.

The selection of $B$ hadron final states will start with an inclusive muon signature (tagging of $b$ production via the semi-leptonic decay of

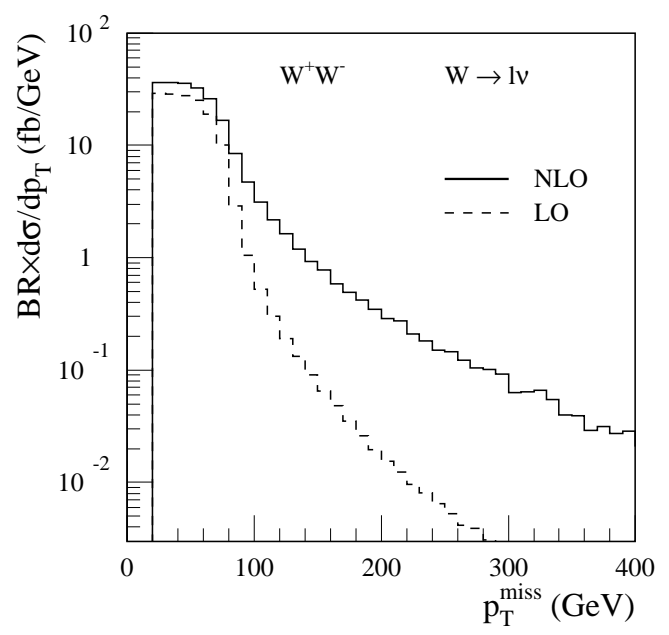

Figure 14. Cross-section for $W^{+} W^{-}$production as a function of the missing transverse momentum $p_{T}^{m i s s}$ (with $W \rightarrow l \nu$, where $l=e, \mu$ ), for leading order (LO) and next-to-leading order (NLO).

a $b$ quark). Depending of the minimal value of the transverse momentum $p_{T}$ of this muon the expected number of events accumulated during three years at low luminosity (corresponding to $30 \mathrm{fb}^{-1}$ ) is shown in figure 16. The number of inclusive $B$ hadrons (identified via a reconstructed $J / \Psi \rightarrow \mu \mu$ decay) is larger by one order of magnitude than the one of exclusive $B$ decays. In the latter case, also the other decay products of $B_{d}, B_{s}, B^{+}$and $\Lambda_{b}$ decays (which involve a $J / \Psi \rightarrow \mu \mu$ in the final state) have to be reconstructed. The expected number of events will allow studies for transverse momenta up to $100 \mathrm{GeV}$ with negligible statistical uncertainties.

\section{Summary}

Studies of QCD processes at the LHC will provide further tests of QCD as the theory of strong interactions at the energy frontier of $14 \mathrm{TeV}$. The expected performance of the ATLAS and CMS detectors will allow in addition precision measure- 


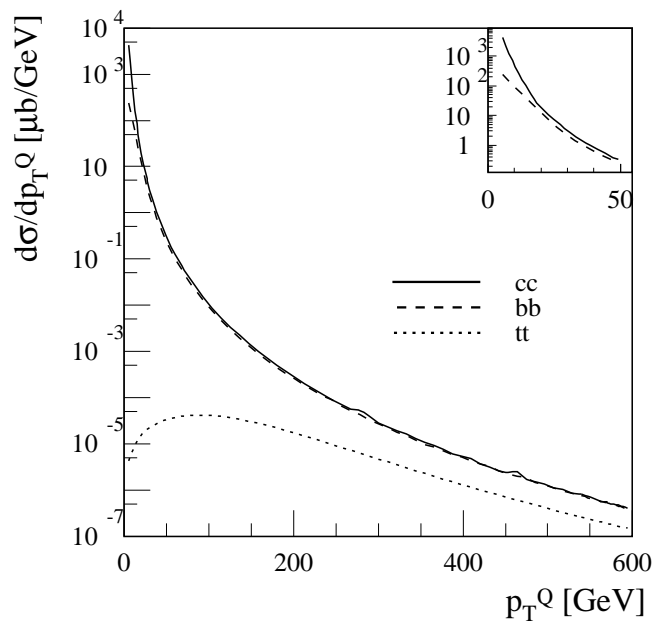

Figure 15. Cross-section for pair production of heavy quarks $(c \bar{c}, b \bar{b}$ and $t \bar{t})$ as a function of the heavy quark transverse momentum $p_{T}^{Q}$.

ments of QCD processes to be performed. These measurements should lead to improvements in the knowledge of the proton structure (in as yet unexplored kinematical regions) and possibly measurements of the strong coupling constant up to the highest scales.

\section{Acknowledgements}

I would like to thank Prof. S. Narison and the organizing committee for the invitation to present this report and for the stimulating atmosphere at the meeting. Furthermore I would like to thank my co-convenors of the QCD working groups of the LHCC workshop on 'Standard Model physics and more' [6] (S. Catani, M. Dittmar, D. Soper and W.J. Stirling) and of the Les Houches workshop on 'TeV collider physics' [7] (S. Catani, J. Huston, M. Dittmar and D. Soper) for interesting discussions.

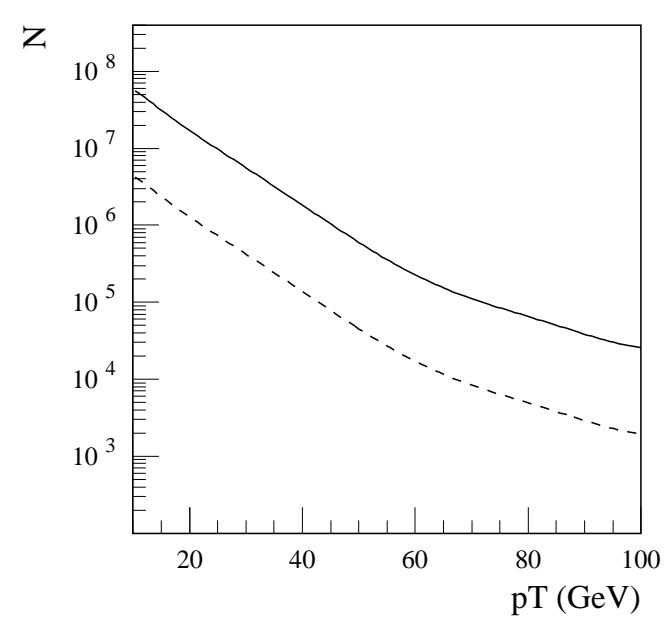

Figure 16. Number of inclusive (solid line) and exclusive (dashed line) B hadron decays as a function of the minimal muon transverse momentum, which should be collected with $30 \mathrm{fb}^{-1}$ of integrated luminosity.

\section{REFERENCES}

1. ATLAS collaboration, Technical Proposal, CERN/LHCC/94-43 (1994).

2. CMS collaboration, Technical Proposal, CERN/LHCC/94-38 (1994).

3. ATLAS collaboration, Detector and Physics Performance Technical Design Report, CERN/LHCC/99-14 (vol. I) and CERN/LHCC/99-15 (vol. II) (1999).

4. R. Bonino et al., Phys. Lett. 211B (1988) 239.

5. TOTEM collaboration, Technical Proposal, CERN/LHCC/99-07 (1999)

6. LHCC workshop on 'Standard Model physics and more' 'http://home.cern.ch/ mlm/lhc99/ lhcworkshop.html'

7. Les Houches workshop on 'Physics at TeV colliders' 'http://lappc-th8.in2p3.fr/houches99/ intro.html' 